\title{
Analysis of Renal Fibrosis in a Rabbit Model of Crescentic Nephritis
}

\author{
Greg Downer, Sem H. Phan, and Roger C. Wiggins \\ Departments of Internal Medicine and Pathology, University of Michigan Medical School, Ann Arbor, Michigan 48109-0602
}

\section{Abstract}

The pathogenesis of renal fibrosis in crescentic nephritis is incompletely understood. To improve our understanding of this process, crescentic nephritis was induced in New Zealand White rabbits by administration of guinea pig antiglomerular basement membrane IgG after sensitization with guinea pig IgG; and their kidneys were analyzed for the development of fibrosis. Collagen synthesis in renal cortical tissue was signifcantly elevated by day 3; peaked at days 7-15, and returned towards baseline by day 21. Collagen content of both glomeruli and cortex were increased starting on days 14-16, and remained constant in cortex thereafter. Light microscopic analysis was much less sensitive, revealing fibrosis only after day 21. Immunofluorescence revealed that type IV collagen was distributed primarily in the glomerulus, while types I and III were increased in the glomerulus and interstitium. Thus, in this model of crescentic nephritis, fibrosis, as assessed biochemically, developed early at time points when morphologic analysis failed to detect such a development. Hence early therapeutic intervention, before morphologic evidence of fibrosis is evident, may be more successful in arresting the progression of this disease before it reaches irreversible terminal stages.

\section{Introduction}

Increased collagen accumulation with progressive renal scarring is the final common pathway to chronic renal failure in many renal diseases. Much has been learned recently about the pathogenesis of glomerular and interstitial fibrosis from the study of animal models (1-5), human renal biopsies (6), and cell culture systems (7-9). However, less has been published regarding the time course for development of renal fibrosis. It has been observed clinically that therapy is less effective in preventing or delaying chronic renal failure once significant fibrosis is present (10). Furthermore, the interaction between elements regulating fibrogenesis may be better understood by looking at the net effect on collagen synthesis and accumulation. The principal aim of this study was to examine histologically and biochemically the development of renal fibrosis in a rabbit model of crescentic nephritis.

From previous reports it appears that both interstitial (I and III) and basement membrane (IV and V) collagens consti-

Address all correspondence to Dr. Sem H. Phan, Department of Pathology M0602, University of Michigan Medical School, Ann Arbor, MI 48109-0602.

Received for publication 30 November 1987 and in revised form 7 March 1988.

J. Clin. Invest.

(c) The American Society for Clinical Investigation, Inc.

0021-9738/88/09/0998/09 \$2.00

Volume 82, September 1988, 998-1006 tute the fibrotic lesion in certain glomerular diseases $(1,5,6)$. Their distribution between the glomerulus and interstitium may be dependent on the integrity of Bowman's capsule (6). Another aim of this study was to examine the pattern of distribution of interstitial and type IV collagens in this animal model.

\section{Methods}

Model of antiglomerular basement membrane disease. The method used to produce crescentic nephritis in rabbits has been previously described (11). Briefly, New Zealand White rabbits, weighing 2.0-3.0 $\mathrm{kg}$, were injected subcutaneously with $100 \mu \mathrm{g}$ of guinea pig IgG in CFA (Sigma Chemical Co., St. Louis, MO) and $5 \mathrm{~d}$ later they were given an intravenous injection of guinea pig anti-rabbit glomerular basement membrane (anti-GBM) ${ }^{1}$ IgG. Control animals received no injections. Rabbits were kept in individual cages and were given water and regular rabbit chow ad lib.

At the indicated times, renal tissue samples were obtained for analysis from these animals. Rabbits were sedated with $25-50 \mathrm{mg} / \mathrm{kg}$, i.v. sodium pentobarbital. The kidneys were then perfused with normal saline until blanched (100 to $200 \mathrm{~cm}^{3}$ ), and then with $180 \mathrm{~cm}^{3}$ of iron oxide $(2.5 \mathrm{mg} / \mathrm{ml})$ in $1 \%$ BSA. The kidneys were then harvested, the capsule was removed, and the cortex was trimmed off with scissors. A piece of cortex was homogenized in $0.5 \mathrm{M}$ acetic acid by polytron (Brinkmann Instruments Co., Westbury, NY), divided into three aliquots, dried in a speed vac concentrator (Savant Instruments, Inc., Hicksville, NY), and stored at $-70^{\circ} \mathrm{C}$. Sections for light microscopy were placed in $10 \%$ formalin, and sections for immunofluorescent analysis were frozen in liquid nitrogen and stored at $-70^{\circ} \mathrm{C}$. The remaining cortex was homogenized in chilled PBS by a Polytron (Brinkman Instruments) and then passed sequentially through a brass screen, 25 openings per inch (Bel-Art Products, Pequannock, NJ), a nylon screen, $90-\mu \mathrm{m}$ opening diam (Tetko Inc., Elmsford, NY), and into a 250-ml beaker. The filtrate was then placed on a magnet and the iron-embolized glomeruli were purified by repeated washings with chilled PBS. Glomerular purity and yield were determined by lowpower $(\times 10)$ examination of $10-\mu 1$ samples. The glomeruli were then divided into three aliquots, suspended in $0.5 \mathrm{M}$ acetic acid, dried in a speed vac concentrator and stored at $-70^{\circ} \mathrm{C}$.

Histologic scoring. All kidney sections were scored by one of the authors (G. Downer) who was blinded to the time-point of disease. The degree of cortical fibrosis was assessed on Masson's trichrome-stained sections and graded 0-3+ (none, mild, moderate, severe) based on the total amount of blue stain throughout the cortex (Sclerosis Score). A minimum of $10 \times 40$ fields were examined per animal. The activity of glomerular involvement was also assessed. Glomeruli were categorized as normal, acutely inflamed (increased cellularity of the tuft, proteinaceous material in Bowman's space or cellular crescents) or chronically diseased (fibrosis involving the glomerular tuft or crescent). For each rabbit, 50 glomeruli were examined and the percentage of glomeruli in each category (normal, acute disease, chronic disease) was recorded.

Collagen content. This was measured as total hydroxyproline $(\mathrm{OH}-$ Pro) by the method of Huszar et al. (12) as modified by Phan et al. (13).

1. Abbreviations used in this paper: GBM, glomerular basement membrane; OH-Pro, hydroxyproline; TBM, tubular basement membrane. 
Briefly, aliquots of glomeruli or cortex were suspended in $6 \mathrm{~N} \mathrm{HCl}$ and hydrolyzed overnight at $110^{\circ} \mathrm{C}$ in tightly capped tubes. The samples were then dried in a speed vac concentrator and resuspended in citrate-acetate buffer ( $\mathrm{pH} \mathrm{6.0)}$. The sample was oxidized by chloramine T (Kodak Co., Rochester, NY), mixed with p-dimethylaminobenzaldehyde, and absorbance at $550 \mathrm{~nm}$ was measured. OH-Pro content was calculated from a standard curve of purified OH-L-Pro (Sigma Chemical Co.). Results were expressed as OH-Pro per glomerulus, per milligram dry weight (for cortex), per milligram protein or per microgram DNA.

DNA content. This was measured by the method of Burton (14) as modified by Phan et al. (15). Briefly, DNA was extracted from samples of glomeruli or cortex by heating in $5 \% \mathrm{TCA}$ at $90^{\circ} \mathrm{C}$ for $15 \mathrm{~min}$. The extract was then mixed with diphenylamine (Sigma Chemical Co.) at $100^{\circ} \mathrm{C}$ for $10 \mathrm{~min}$ and absorbance at $600 \mathrm{~nm}$ was measured. DNA content was calculated from a standard curve of purified calf-thymus DNA (Sigma Chemical Co.).

Total protein. This was determined by the Lowry protein assay (16).

Collagen synthesis. The method of Phan et al. for measuring the rate of lung collagen synthesis was adapted to kidney tissue with minor modifications (17). Briefly, New Zealand White rabbits were immunized as described above, sedated with sodium pentobarbital at the time of their death, and the kidneys were perfused with normal saline until blanched. Slices of fresh cortex (100-200 mg wet weight) were then minced with scissors into 1-2-mm pieces and incubated in $2 \mathrm{ml}$ of media containing sodium ascorbate $(50 \mu \mathrm{g} / \mathrm{ml}), \beta$-aminopropionitrile $(80 \mu \mathrm{g} / \mathrm{ml})$, and $10 \mu \mathrm{Ci}\left[{ }^{3} \mathrm{H}\right]$ proline $(100 \mathrm{Ci} / \mathrm{mmol})$ (ICN Radiochemicals, Inc., Irvine, $\mathrm{CA}$ ) for $5-6 \mathrm{~h}$ at $37^{\circ} \mathrm{C}$, in $95 \% \mathrm{O}_{2}, 5 \% \mathrm{CO}_{2}$. After washing the tissue fragments with PBS to remove unincorporated $\left[{ }^{3} \mathrm{H}\right]$ proline, they were suspended in $10 \%$ TCA and homogenized with a Polytron. DNA content was determined on an aliquot of the homogenate. The rest of the sample was washed twice with $10 \%$ TCA and resuspended in $0.1 \mathrm{M} \mathrm{NaOH}$. The sample was then neutralized by the addition of $1 \mathrm{M}$ Tris- $\mathrm{HCl}(\mathrm{pH} 7.3$ ) and titrated to $\mathrm{pH} 7.2-7.7$ with $1 \mathrm{M}$ $\mathrm{HCl}$. The sample was then digested with purified bacterial collagenase (Worthington Biochemical Corp., Freehold, NJ) at $37^{\circ} \mathrm{C}$ for $2 \mathrm{~h}$. The digestion was stopped with $20 \%$ TCA and $0.5 \%$ tannic acid and the samples were counted in Safety-solve (Research Products International Corp., Mount Prospect, IL) using a liquid scintillation counter (Beckmann LS 5801; Beckmann Instruments Inc.). The collagenase-liberated counts (TCA soluble) represented collagenous proteins and the collagenase-undigested precipitate represented noncollagenous proteins. The specific radioactivity of tissue proline pools was determined on the pooled TCA wash supernates and were used to convert disintegrations per minute to nanomoles of proline incorporated. Results were expressed as nanomoles proline incorporated per gram dry weight (\% control) or per milligram DNA (\% control).

Antiserum to types I and III collagen. Interstitial collagens (I and III) were isolated from rabbit lungs using the method of Seyer et al. (18). Rabbit lungs (Pel-Freez Biologicals, Rogers, AR) were trimmed of extraneous lung tissue and were washed twice with PBS. They were then minced into $\sim 2-\mathrm{mm}$ pieces, resuspended in $0.5 \mathrm{M}$ acetic acid, and homogenized with a Polytron. Pepsin was then added to a final concentration of $0.5 \mathrm{mg} / \mathrm{ml}$ (Sigma Chemical Co.) and the suspension slowly stirred at $4^{\circ} \mathrm{C}$ for $24 \mathrm{~h}$. Upon centrifugation, the supernate was stored frozen, while the pellet was reextracted twice with the acetic acid/pepsin solution. All extracts were then pooled and neutralized to pH 7.6 with $1 \mathrm{M}$ Tris. $\mathrm{NaCl}$ was added to a final concentration of 4.5 $M$ to precipitate collagen, then the solution was centrifuged. The pellet was resuspended in $0.5 \mathrm{M}$ acetic acid and dialyzed against $0.5 \mathrm{M}$ acetic acid containing $0.7 \mathrm{M} \mathrm{NaCl}$. After centrifugation, the pellet containing types I and III collagen was dialyzed against $50 \mathrm{mM}$ Tris- $\mathrm{HCl} \mathrm{pH} 7.5$, and $1 \mathrm{M} \mathrm{NaCl}$. The $\mathrm{NaCl}$ concentration was then raised to $1.7 \mathrm{M}$, and the sample was centrifuged to separate the type I (pellet) from type III collagen (supernate). This fractionation was repeated twice to minimize contamination by type III collagen in the type I collagen pellet. The final preparation was at least $95 \%$ type I collagen with $<5 \%$ contamination by type III collagen as quantitated by SDS-PAGE with interrupted reduction (19). No other contaminant was apparent by SDS-PAGE.

A female goat was immunized subcutaneously with $10 \mathrm{mg}$ (dry weight) of this collagen preparation in $1 \mathrm{~cm}^{3} \mathrm{CFA}$ and was given three booster injections biweekly of $2 \mathrm{mg}$ collagen in $1 \mathrm{~cm}^{3}$ CFA subcutaneously. $7 \mathrm{~d}$ after the final injection the goat was exsanguinated, and the serum was aliquoted and stored at $-30^{\circ} \mathrm{C}$. This immune serum is hereafter designated anti-I/III, and was absorbed with rabbit serum before use. Western blot analysis using the purified collagen and commercially available pure types I and III collagen (Sigma Chemical Co.) showed staining of predominantly type I collagen bands, but also some type III staining.

Antiserum to type IV collagen. A female goat was immunized with $1 \mathrm{mg}$ of purified human placenta type IV collagen (Sigma Chemical Co.) in $1 \mathrm{~cm}^{3}$ CFA subcutaneously for a total of seven doses at biweekly intervals. $7 \mathrm{~d}$ after each of the last two immunizations, 100-200 $\mathrm{ml}$ of blood was obtained by jugular vein puncture, the serum was pooled, aliquoted, and stored at $-30^{\circ} \mathrm{C}$. Western blot analysis of plasma-absorbed antibody showed staining of type IV human collagen.

Affinity chromatography. In some studies anti-type I/III and antitype IV antibodies were immunopurified by passage of immune IgG fractions over Sepharose 4B columns to which type I/III or type IV collagen had been immobilized by cyanogen bromide activation (20). Briefly, purified rabbit type I/III or human placental type IV (Sigma Chemical Co.) collagen were immobilized onto Sepharose 4B after cyanogen bromide activation (20). After inactivation of unreacted sites, the beads were poured into a $1.0 \times 10-\mathrm{cm}$ column, and IgG fraction of anti-type I/III or anti-type IV antiserum were then applied. The columns were then eluted with at least two column volumes of PBS until no more $280 \mathrm{~nm}$ absorbing material was detected in the effluent. Specific antibodies were then eluted from the affinity column by glycine- $\mathrm{HCl}$ buffer, $\mathrm{pH} 2.0$. No differences were observed in the immunofluorescent patterns using affinity-purified antibodies compared with unfractionated antiserum.

Immunofluorescence. Indirect fluorescence using goat anti-serum against interstitial (anti-I/III) or type IV collagens (anti-IV) diluted 1:100, or affinity-purified IgG, was done using fluorescein-labeled rabbit anti-goat IgG (Cappel Scientific Division, Cooper Biomedical, Inc. Malvern, PA) as the second antibody. Normal goat serum was used as the control. Fluorescence was viewed and photographed with a Zeiss photomicroscope (Morgan Instruments, Inc., Detroit, MI).

Statistics. All results in experimental groups were compared with control values using the unpaired Student's $t$ test to examine for signifcant differences $(P<.05)$.

\section{Results}

Histologic changes. The changes in glomerular morphology previously described in this model were confirmed in the present study (Fig. 1) (11). There was increased cellularity of the tuft by days 3-5 and proteinaceous material in Bowman's space by day 7 , which was followed by cellular crescents (days 10-14), and diffuse glomerular and interstitial fibrosis (days 21-35).

In Fig. 2 the percentage of involvement of glomeruli by acute or chronic changes in relation to time is shown. By days $6-9,90 \%$ of glomeruli have acute inflammatory changes. At days $51-55$, however, only $3 \%$ of glomeruli are inflamed as defined by increased numbers of cells or proteinaceous matrix, while $47 \%$ of glomeruli appear normal and $50 \%$ show fibrosis.

Isolation of glomeruli. Purity of the glomerular preparations for the control, and in the days-6-9, and days-14-16 groups was $84 \pm 4 \%, 87 \pm 5 \%$, and $79 \pm 3 \%$, respectively. The glomeruli isolation procedure resulted in our obtaining prepa- 

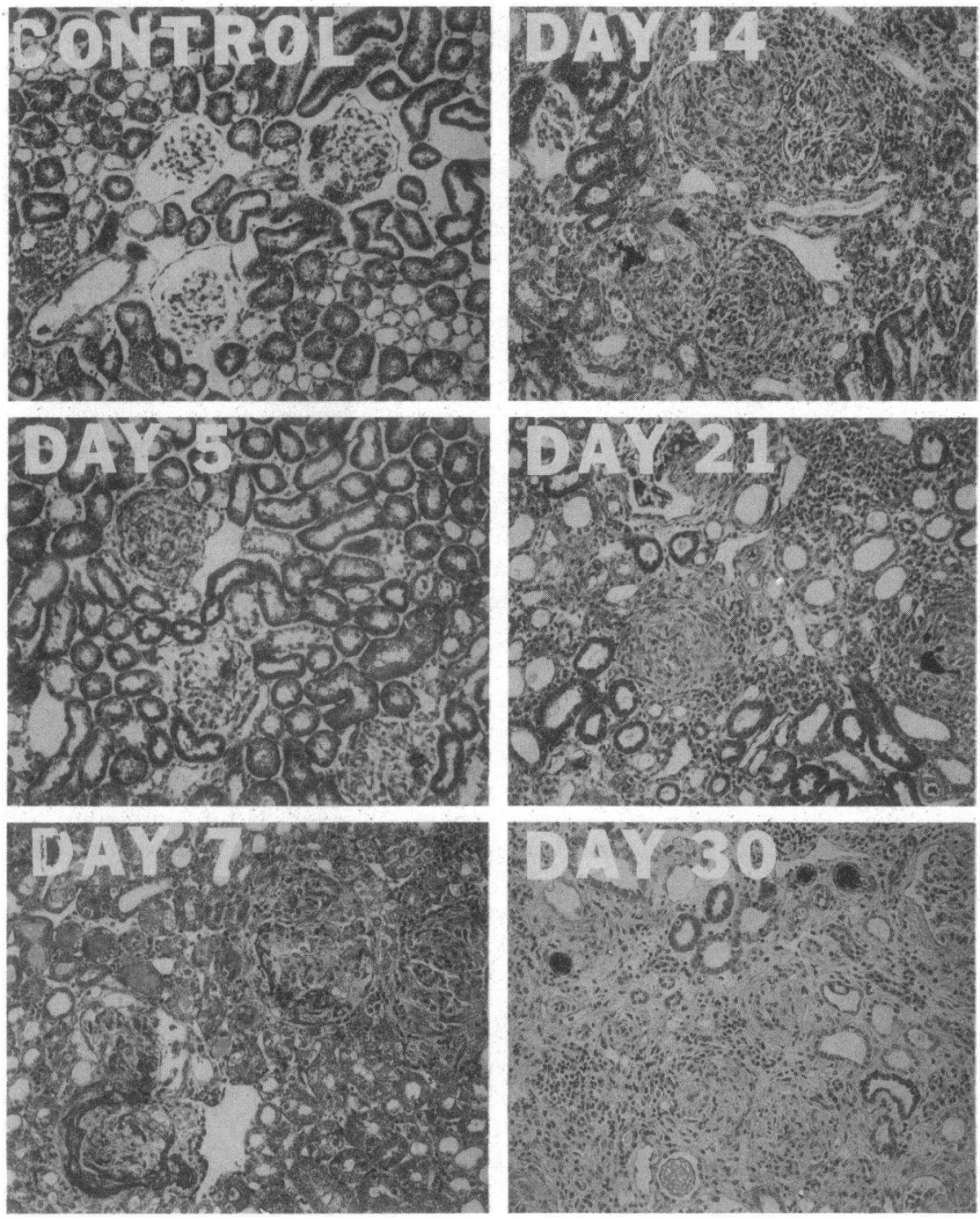

Figure 1. Masson trichrome-stained histological sections of kidney from rabbits. The dark homogeneous areas within glomeruli and vessels are the iron oxide perfused into the kidney to facilitate glomerular purification. Note the increase in number of cells per glomerulus on day 5 , the formation of fibrin-containing proteinaceous material in Bowman's space on day 7 , the cellular crescent and periglomerular interstitial mononuclear infiltrate on day 14 , the homogeneous appearance of some glomeruli indicating replacement by collagenous matrix (blue-stained), and the loss of tubules by day 21 , and the widespread fibrotic process by day 30. rations that were biased towards more normal-appearing glomeruli. This is illustrated by the results from a separate study (Eldredge, Wiggins, and Kunkel, unpublished data), where the morphologic appearance of isolated glomeruli was compared with the morphologic appearance of glomeruli in histological sections from the same animals. Glomeruli in each case were scored as normal, increased number of cells, or crescents present. The values given as the percentages of total number of normal, increased cells or crescentric glomeruli for isolated glomeruli/histological sections at day 4 were $16 \pm 4 / 8 \pm 2$, $84 \pm 8 / 92 \pm 6$, and $0 / 0$; for day 7 they were $30 \pm 7 / 4 \pm 2$, $42 \pm 8 / 46 \pm 7$, and $28 \pm 3 / 50 \pm 9$; for day 14 they were $59 \pm 10 /$ $14 \pm 5,14 \pm 6 / 26 \pm 8$, and $27 \pm 4 / 60 \pm 10$. For day $21>95 \%$ of isolated glomeruli appeared normal compared with $\sim 50 \%$ in the histological sections. Therefore, we were not able to isolate representative glomeruli after day 14 of the disease model using the iron embolization technique, and even those glomeruli isolated from before day 21 were significantly biased towards more normal glomeruli. Thus, the data for isolated glomeruli must be interpreted with this bias in mind.

Collagen accumulation in glomeruli and cortex. Glomerular OH-Pro content was increased significantly by days 14-16 (Fig. 3) when expressed as OH-Pro per glomerulus (2.30 \pm 0.63 ng OH-Pro/glomerulus vs. control $0.77 \pm 0.12$ ng OH-Pro/glomerulus, $P<.05)$, or as OH-Pro per unit DNA $(0.19 \pm 0.04 \mu \mathrm{g}$ $\mathrm{OH}-\mathrm{Pro} / \mu \mathrm{g}$ DNA vs. control $0.078 \pm 0.013 \mu \mathrm{g} \mathrm{OH}-\mathrm{Pro} / \mu \mathrm{g}$ DNA, $P<.05)$. DNA and total protein per glomerulus were also increased although not significantly. This latter result is not in accordance with the histological data and probably re-

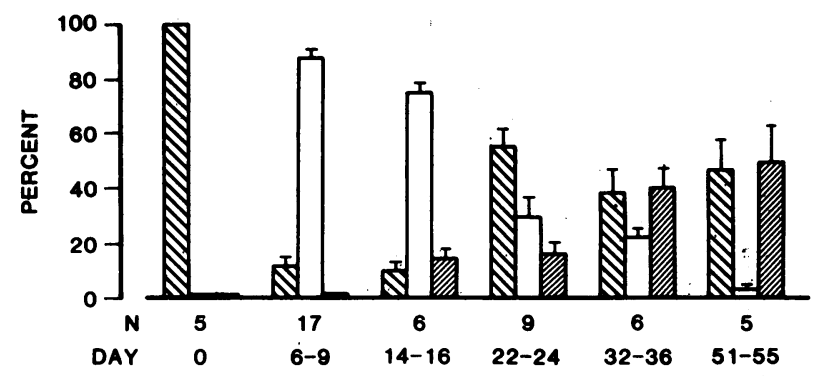

Figure 2. Time-course of glomerular histology in the model of antiGBM disease. Glomeruli are scored as normal (\$), inflamed (containing increased number of cells or fibrin-containing proteinaceous material) ( $\square$ ), or scarred (stained blue with Masson trichrome) (四). $N$, the number of animals used per group. 
Figure 3. Measurements of OH-PRO, DNA, and protein content in glomeruli isolated from control, days 6-7, and days 14-16 animals. Note that the DNA per glomerulus did not rise significantly by day 14 , suggesting that the isolated population of glomeruli was skewed towards the more normal glomeruli and/or that cellular or proteinaceous casts were lost during the isolation procedure. This was confirmed by examining $1-\mu \mathrm{m}$ sections of methacrolate-embedded isolated glomeruli (data not shown). In spite of this bias, glomerular OH-PRO increased by day 14 . ( ${ }^{*} P$ $<0.05$ compared with control or days 6-9 glomeruli). The number of animals $(N)$ examined in each group is indicated inside the bars.

flects the failure to isolate representative glomeruli on day 14 or the loss of cellular crescents from the isolated glomeruli. In spite of this, the OH-Pro content per glomerulus was increased by day 14 .

OH-Pro content in the renal cortex (Fig. 4) was significantly increased by days 6-9 when expressed per unit DNA $(0.141 \pm 0.013 \mu \mathrm{g} \mathrm{OH}-$ Pro $/ \mu \mathrm{g}$ DNA vs. control $0.095 \pm 0.006 \mu \mathrm{g}$

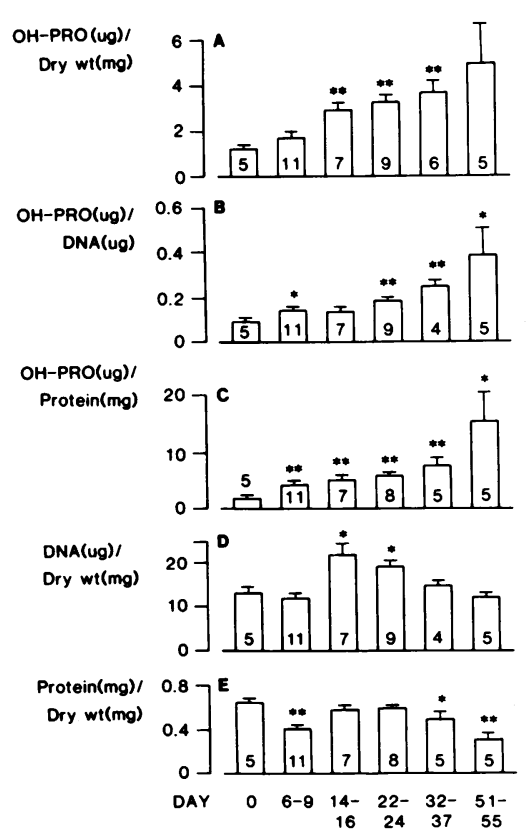

Figure 4. Renal cortical measurement of $\mathrm{OH}$ PRO, DNA, protein, and dry weight at various times during evolution of crescents in rabbit model of anti-GBM disease. $\left({ }^{*} P<0.05 ;{ }^{* *} P\right.$ $<0.01$ when compared with control cortex).

The number of animals used per group is shown inside the bars.

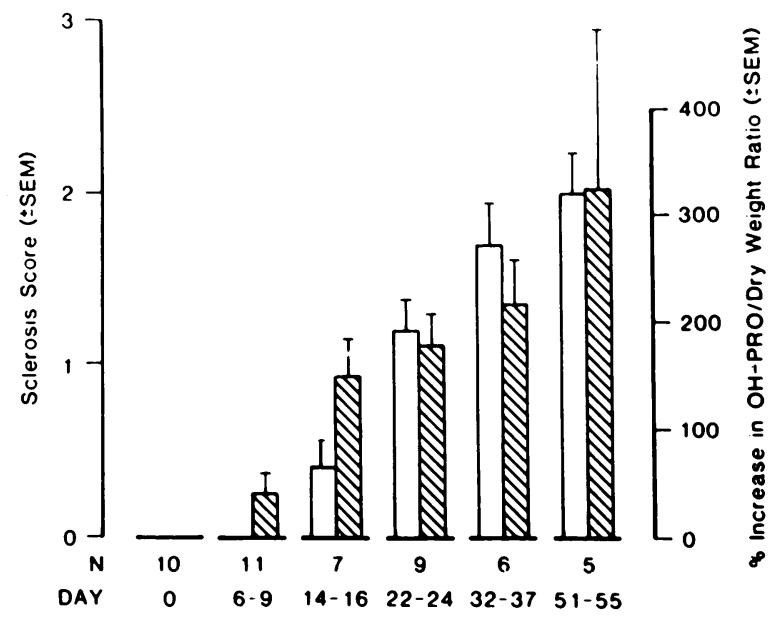

Figure 5. Relationship between the cortical sclerosis score as assessed by Masson trichrome staining (glomerulus and interstitial scarring) (ㅁ), and the measured cortical OH-PRO expressed per dry weight $(\bowtie)$. Note that Masson trichrome staining lags behind collagen accumulation until day 21 . Therefore Masson trichrome staining is not a good index of early collagen synthesis in the kidney. $N$, the number of animals used per group.

OH-Pro $/ \mu \mathrm{g}$ DNA, $P<.05)$ and per unit protein $(4.22 \pm 0.50 \mu \mathrm{g}$ $\mathrm{OH}-\mathrm{Pro} / \mathrm{mg}$ protein vs. control $1.79 \pm 0.20 \mu \mathrm{g} \mathrm{OH}-\mathrm{Pro} / \mathrm{mg}$ protein, $P<.01$ ). It was increased by days 14 to 16 when expressed per dry weight $(2.91 \pm 0.41 \mu \mathrm{g} \mathrm{OH}-\mathrm{Pro} / \mathrm{mg}$ dry weight vs. control $1.18 \pm 0.11 \mu \mathrm{g}$ OH-Pro/mg dry weight, $P$ $<.01$ ). Cortical OH-Pro increased insignificantly thereafter although it remained significantly higher than controls. DNA in the cortex was significantly elevated at days 14-16 (21.8 \pm 2.4 $\mu \mathrm{g} \mathrm{DNA} / \mathrm{mg}$ dry weight vs. control $12.7 \pm 1.7 \mu \mathrm{g} \mathrm{DNA} / \mathrm{mg}$ dry weight, $P<.05$ ) and returned to baseline by days $32-37$ $(14.6 \pm 0.9 \mu \mathrm{g} \mathrm{DNA} / \mathrm{mg}$ dry weight, $P<.05)$, paralleling the increased cellularity seen histologically. The degree of cortical

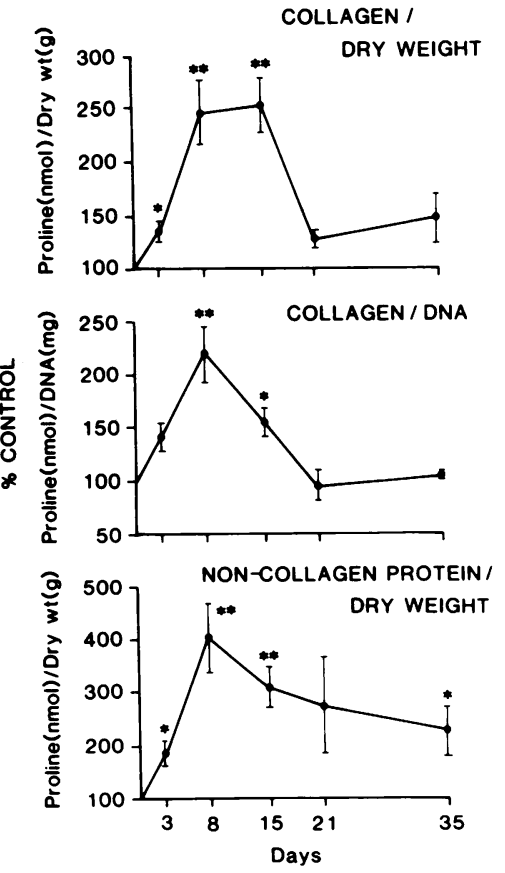

Figure 6. Collagen synthetic rate as assessed by $\left[{ }^{3} \mathrm{H}\right]$ proline incorporation into tissue slices and expressed per dry weight and per DNA. Note that both collagen and noncollagen protein synthesis increased by day 3 , which shows that collagen synthesis was part of a general increase in protein synthesis. Five animals per time point were used to generate this graph. $\left({ }^{*} P\right.$ $<0.05 ;{ }^{* *} P<0.001$ when compared with control synthetic rate). 


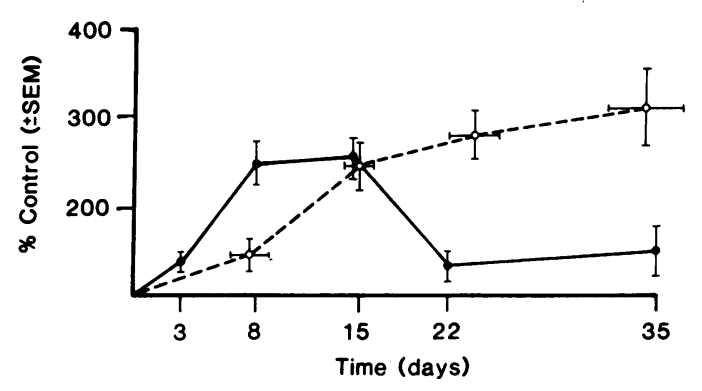

Figure 7. Time-course of collagen synthetic rate (expressed per dry weight) (-) in relation to collagen accumulation (expressed per dry weight) (- - ). The horizontal error bars represent the range of days at which measurements were made. The vertical error bars represent the SE of the mean. Note the time lag between the increase in collagen synthetic rate and the accumulation of collagen in renal cortex. Five animals per time point were used to generate the collagen synthetic rate data. The number of animals used for the collagen accumulation data is as shown in Fig. $4(A)$.

fibrosis on Masson's trichrome-stained sections underestimated the increase measured biochemically at early time points. At days 14-16 when cortical OH-Pro had increased by $150 \%$, there was only mild fibrosis histologically (Fig. 5).

Collagen synthesis. The rate of synthesis of collagen in the cortex (Fig. 6) was significantly elevated by day 3 when expressed per dry weight $(135 \pm 9 \mathrm{nmol}$ proline/g dry weight, \% control, $P<.05)$, peaked around day $15(255 \pm 24 \mathrm{nmol}$ pro- line/g dry weight, \% control, $P<.001)$, and returned to control levels by day 21 (127 $\pm 9 \mathrm{nmol}$ proline/g dry weight, \% control, $P<.05$ ). Noncollagen protein synthesis followed a similar pattern. Collagen accumulation and rate of synthesis are directly compared in Fig. 7. As expected, increased collagen content lagged several days behind the increase in rate of synthesis and was maintained after the rate of synthesis returned to baseline.

Patterns of accumulation of interstitial and type IV collagen. Control glomeruli had mesangial and capillary loop staining with anti-type IV collagen antibody. When using anti-type I/III collagen antibody some control glomeruli were negative while others showed scattered mesangial staining (Fig. 8). There was a progressive increase in glomerular fluorescence at days 7 and 14 with both anti-type I/III and anti-type IV. However, the patterns of staining with the two antisera differed. With anti-type IV, fluorescence was increased in the tuft by day 7 although the glomeruli architecture remained intact. By day 14 sheets of homogeneous staining were present in glomeruli and also involved the crescent and immediate periglomerular region (Figs. 8 and 9). Interstitial involvement was limited to brighter and apparent thickening of the tubular basement membrane (TBM). Fluorescence with anti-type I/III, on the other hand, involved both the glomerulus and interstitium. At day 7, tuft fluorescence was increased (Fig. 8). In many glomeruli at this time there was also interstitial staining around glomeruli (not shown). At 2 wk, fluorescence in both the glomerulus and the interstitium was further increased (Fig. 8). Interestingly, in some areas there was bright interstitial

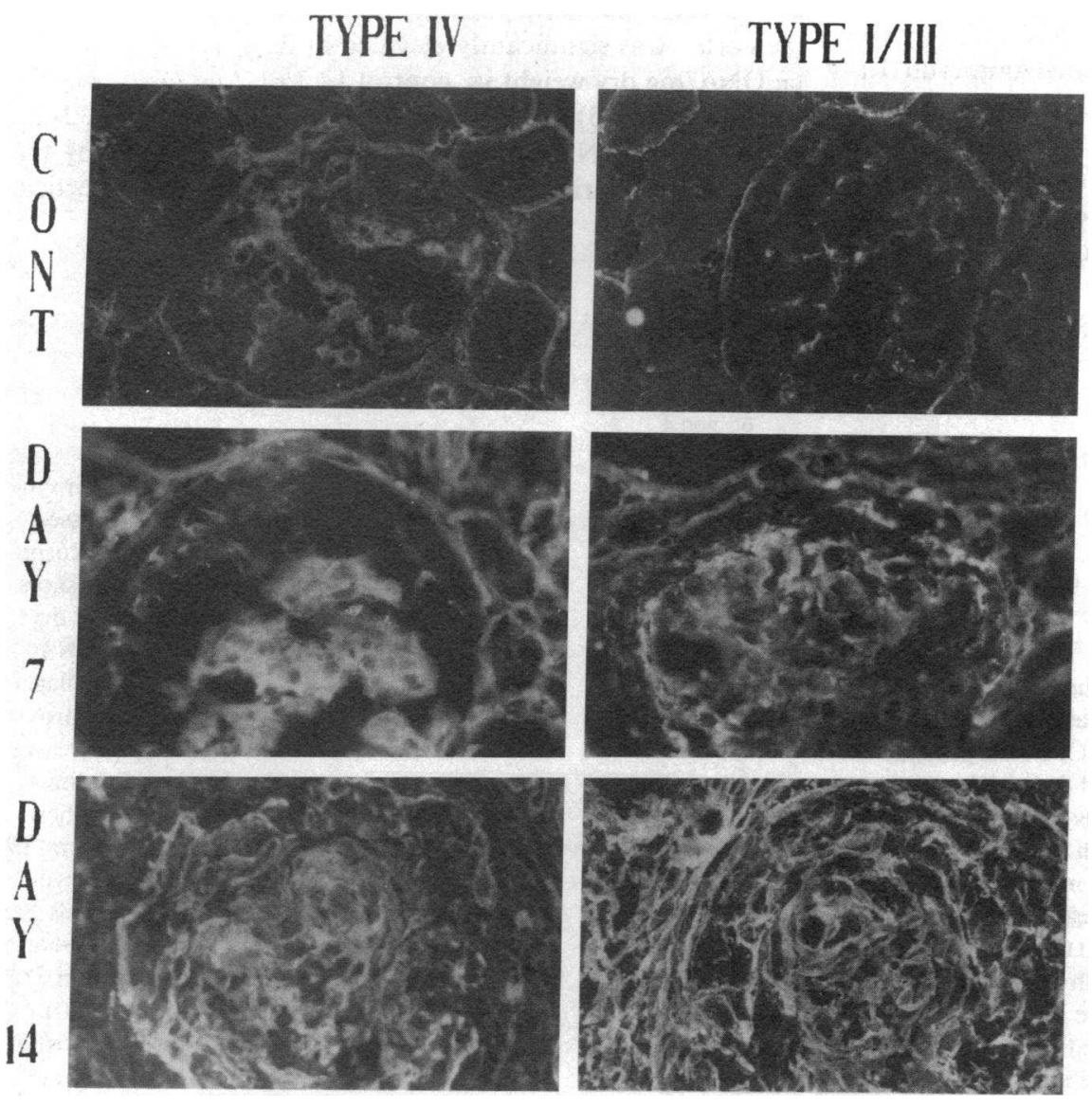

Figure 8. Time-course of type IV and type I/III collagen distribution as assessed by immunofluorescence. Note that by day $7 \mathrm{immu}$ nofluorescence was obviously increased for both type IV and type I/III although the basic glomerular structure appeared to remain relatively intact. By day 14 glomerular architecture was grossly distorted as assessed by both type IV and I/III staining. However, the type I/III staining was much less within glomeruli than in the periglomerular and interstitial areas (see Fig. 10), while the type IV staining was predominantly intraglomerular. 

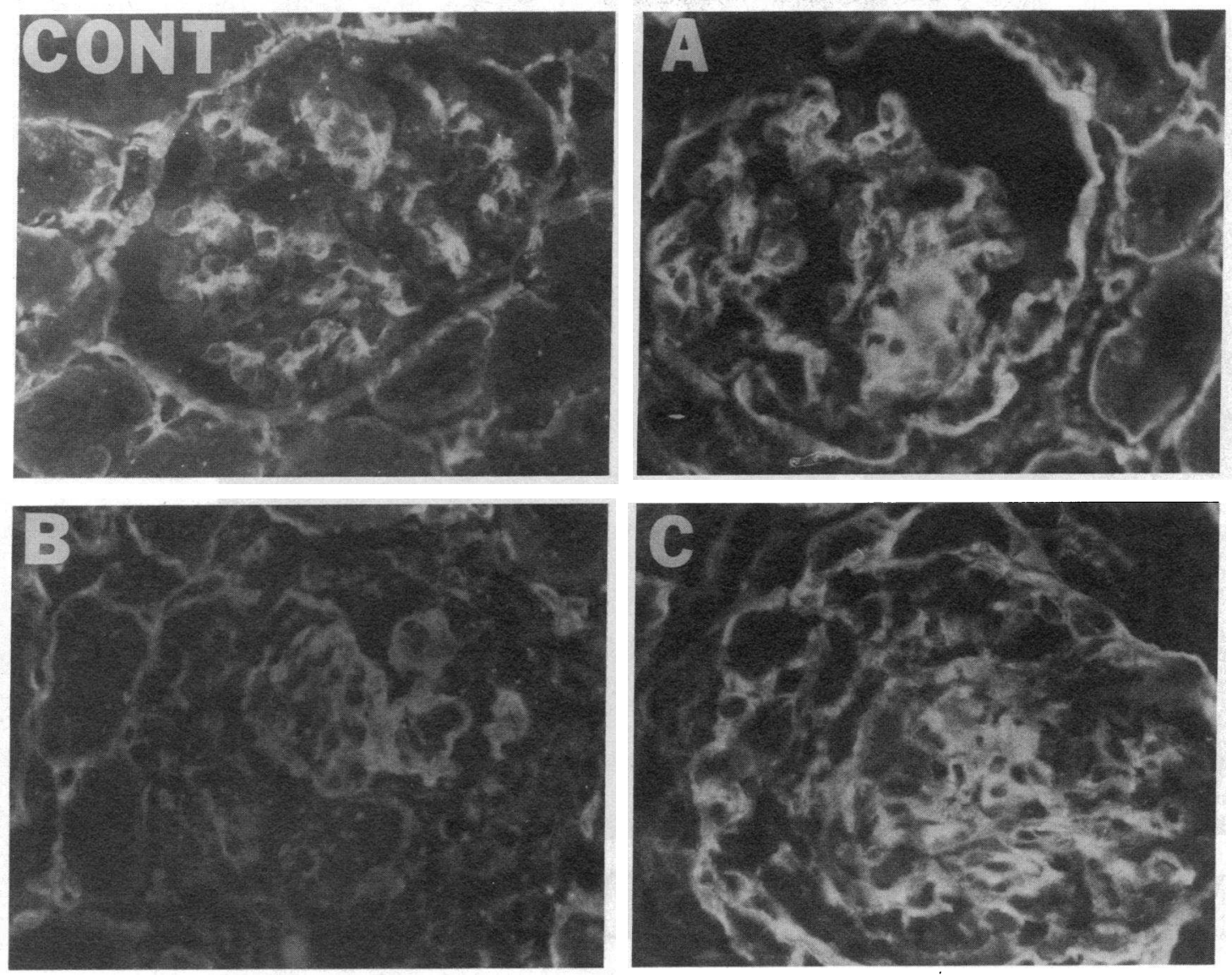

Figure 9. Immunofluorescent sections stained for type IV collagen, in a control animal and animals at day $14(A, B$, and $C)$. Note that by day 14 there are plaques of type IV collagen that may involve parts of glomeruli $(A)$, the whole glomerulus $(B)$, or extend outwards into the interstitial compartment from glomeruli $(C)$.

fluorescence surrounding nonstaining glomeruli (Fig. 10), while in other areas the glomerulus was positive and the surrounding interstitium was negative (not shown).

In summary, anti-type IV staining is localized to the tuft, immediate periglomerular area, and crescent. Fluorescence with anti-type I/III may involve primarily the glomerulus, interstitium, or both.

\section{Discussion}

The pathogenesis of renal fibrosis as a consequence of acute glomerulonephritis is incompletely understood. Collagen-producing cells in the renal cortex include at least epithelial, endothelial, and mesangial cells of the glomerulus and interstitial fibroblasts. While epithelial and endothelial cells make basement membrane collagens (types IV and V) and fibroblasts make interstitial collagens (types I and III), mesangial cells can produce both classes of collagen (types I, III, IV, and V), at least in tissue culture $(8,9,21)$. An array of chemical mediators produced by resident and infiltrating cells have been identified that may influence the rate of collagen synthesis and breakdown $(2,4,7,19,22-26)$. To better understand the regulation of renal fibrosis we studied the time-course of collagen formation in a rabbit model of crescentic nephritis.

In this model, the outcome of the acute inflammatory phase is not uniform among the glomeruli. While $90 \%$ of glo- meruli are involved at day 7, 50\% ultimately become histologically normal again, and $50 \%$ develop fibrosis. The local factors determining these different outcomes are not known.

Fibrogenesis as defined by increased renal cortical collagen synthesis appears to be switched on and then off in the first 2 wk after anti-GBM administration. The rate of collagen synthesis is increased by day 3-4 and rises toward a maximum rate by day 7 , at which time little collagen accumulation has occurred. By $14 \mathrm{~d}$ the synthetic rate is still high, and collagen content has significantly increased in both the glomeruli and the cortex. At $21 \mathrm{~d}$, the rate of synthesis has returned towards baseline although collagen content continues to rise slowly thereafter. While such data are not available for other models of renal disease, a similar pattern was observed in a rat model of pulmonary fibrosis (17). In both cases there is a prompt increase in collagen synthetic rate which lasts for a finite period, followed a few days later by increases in total collagen deposition. This suggests that if the new collagen being synthesized is associated with a rise in collagenolysis, the increase in collagenolysis must be of a smaller magnitude than the increase in synthetic rate. It is possible that the situation in the kidney may be similar to that seen in the fibrotic lung where there is a significant decrease in collagenolytic activity (down to undetectable levels) at time points greater than $1 \mathrm{wk}$ (17). In the present study, although synthetic rate returned to close to normal levels at time points greater than $14 \mathrm{~d}$, there was still a 

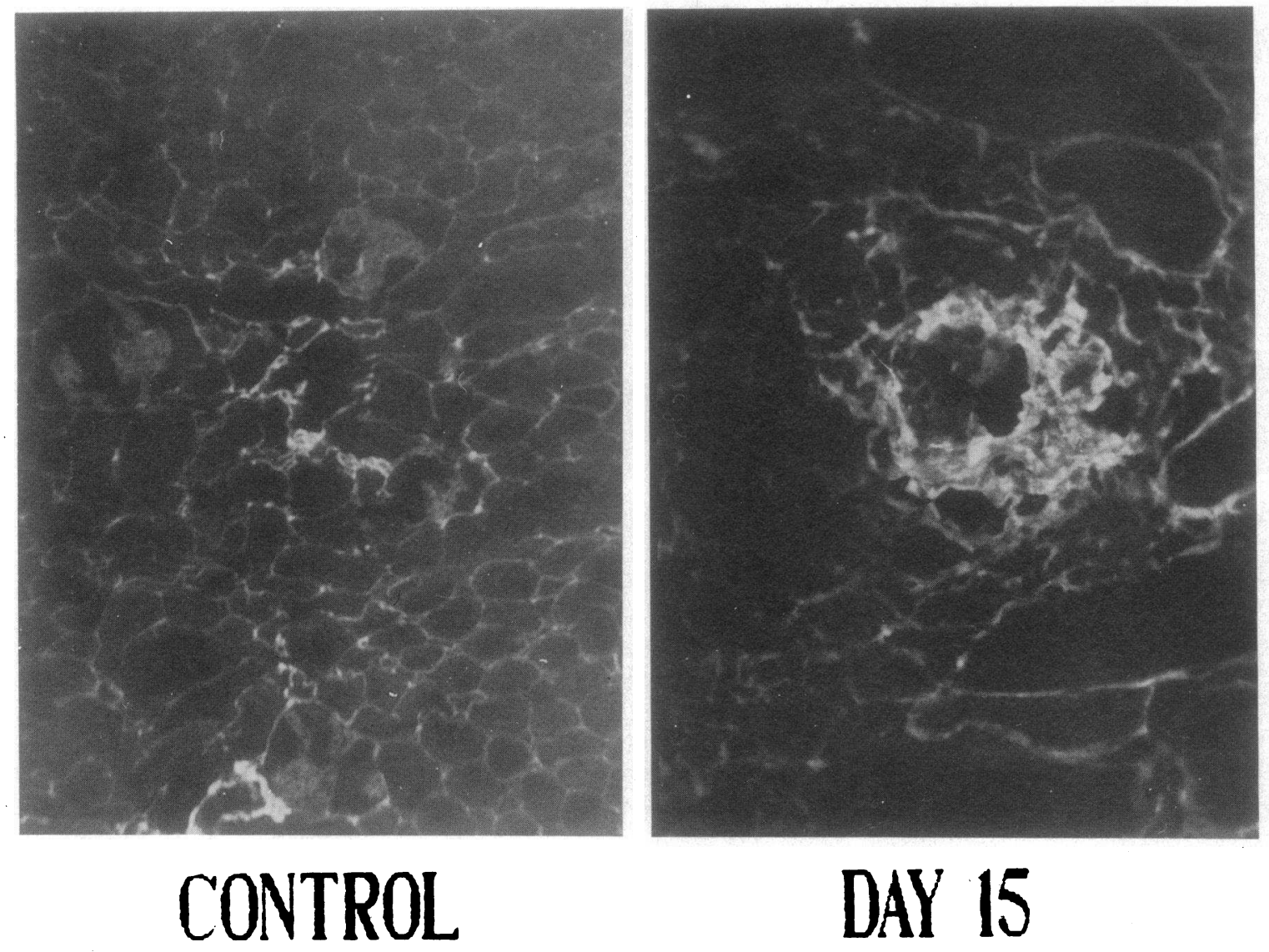

Figure 10. Immunofluorescent type I/III staining of a control and a day 15 animal. Note the marked periglomerular staining at day 15 . In control animals staining with type I/III was predominantly periarteriolar and along TBMs.

small (but not statistically significant) increase above control levels at these later time points. This may account for the continuing, though slight, increase in total collagen content at time points greater than $22 \mathrm{~d}$.

Cell-mediated immunity may play a key role in turning the fibrogenic response on and off (2-4). In a guinea pig model of anti-TBM interstitial nephritis, $T$ lymphocytes early in the disease promote fibroblast proliferation and collagen production (2). By day 18 , however, $T$ lymphocytes begin elaborating a substance that inhibits fibroblast proliferation (2). Thus, while the regulation of fibrogenesis is complex and poorly understood, in these models there is a period of enhanced collagen production after the stimulus, which is followed by downregulation back to control levels.

When comparing histologic and biochemical methods of assessing fibrosis, it becomes apparent that Masson's trichrome staining is relatively insensitive. At $14 \mathrm{~d}$, when cortical OH-Pro has increased by $150 \%$, there is only a mild degree of fibrosis by light microscopy. Furthermore, immunofluorescent analysis showed marked distortion of glomerular architecture with sheets of material staining for type IV collagen when no such changes were seen by Masson trichrome staining. The clinical implication is that kidney biopsies showing even mild fibrosis by Masson trichrome staining may represent a significant increase in cortical collagen accumulation. This would explain in part why individuals with lupus nephritis, for example, with mild fibrosis on biopsy are at high risk for developing chronic renal failure (10), and it raises the question of whether more sensitive indicators of increased collagen synthesis should be used in the assessment of renal biopsy material. On a routine basis, the use of periodic-acidSchiff stain silver methenamine, and van Gieson stains may improve sensitivity for collagen staining somewhat. However, immunofluorescence using collagen antibodies may be preferable because of its sensitivity and specificity as we have shown to exist in this study.

Previous studies have examined the distribution of interstitial and basement membrane collagens in crescentic nephritis. In a rat model of nephrotoxic nephritis, types IV and $\mathrm{V}$ collagen were deposited in the tuft and crescent while interstitial collagens were identified in the tuft (type I), crescent, and interstitium (1). In a study of human renal biopsies, those with crescentic disease showed only type IV in the glomerular tuft while type I and/or type III were identified in the crescent (6). It was postulated that the integrity of Bowman's capsule may have determined the collagen composition of the crescent. In the present study, type IV collagen contributed to glomerular and crescentic fibrosis and to the apparent thickening of TBMs. Interstitial collagen was also found in the glomerulus and crescent but predominated in the interstitial scarring. In this study we did not examine the integrity of Bowman's capsule, so that no conclusions can be drawn regarding the relationship between the increased fluorescence for type I/III collagen and holes in Bowman's capsule. It is inter- 
esting that trace amounts of interstitial collagen were seen in some glomeruli of control animals. Others have reported that types I and III collagen are not seen in the normal glomerulus $(1,5,6)$. However, the results from the control glomerulus stained with monoclonal anti-type I antibody in one of these studies (1) appears to be very similar to our control anti-type I/III results, raising the question of whether some glomeruli of normal animals contain small amounts of interstitial type collagen. While this trace positivity with anti-type I/III in control glomeruli may be due to antiserum impurities or to artifact, the available data do not allow us to rule this out as a real observation. Mesangial cells have been shown to produce types I and III collagen in culture $(8,9)$. This area merits further investigation to establish whether interstitial collagen in glomerular lesions results only from fibroblast infiltration or if it can originate from resident glomerular cells as well.

The potential clinical relevance of these observations can be seen by comparing these studies with our previous studies using the same model. They have shown that monocytes and activated T cells are first seen within glomeruli by day 3 (27, 28 ), proteinuria begins on day 4 , the urine sediment becomes abnormal on days 5-6, oliguria occurs on days 6 and 7 (29, 30 ), and the serum creatinine is raised by day 7 and continues to rise through day 14 (unpublished observation). Thus increased collagen synthesis is seen within $24 \mathrm{~h}$ of influx of monocytes and $\mathrm{T}$ cells, as proteinuria begins, and before the elevation of serum creatinine. Collagen accumulation begins as the serum creatinine first becomes elevated, and, in this model, is largely complete by day 14 . By day 14 , although the Masson trichrome stain is unimpressive for collagen, immunofluorescence shows what are likely to be irreversible structural changes in and around the glomeruli. By the time the Masson trichrome stain is obviously positive collagen synthesis has switched off and little more collagen accumulation occurs.

These findings cannot be directly compared with most cases of human crescentic nephritis because this model is synchronous (all glomeruli moving through the "stages" (11) of crescent formation together), whereas many cases of crescentic nephritis in man are "asynchronous" (glomeruli are at different "stages" of crescent formation' at any one point in time). However, for a single glomerulus the time-course described is probably appropriate and is similar to that previously described in a rat model of crescentic glomerulonephritis (31).

The implications for therapy are therefore that there is a window of opportunity for therapy, but that it is short. Starting theräpy must be an urgent matter where $24 \mathrm{~h}$ lost may count towards permanent loss of renal function. Even a small change in renal function may indicate significant collagen synthesis and accumulation. Finally, in our efforts to improve the prognosis of individuals with rapidly progressive glomerulonephritis (and other forms of inflammatory renal disease) it may be as profitable to focus on reducing the time interval between presentation and treatment (i.e., education) as it will be to develop new forms of antiinflammatory therapy.

\section{Acknowledgments}

This work was supported by National Institutes of Health grants DK-38149, DK-39255, HL-28737, AM-30673, and a grant-in-aid from the American Heart Association and its Michigan Affiliate. Dr.
Phan and Dr. Wiggins are Established Investigators of the American Heart Association.

The authors gratefully acknowledge the expert technical assistance of Bridget McGarry.

\section{References}

1. Foellmer, H. G., R. B. Sterzel, and M. Kashgarian. 1986. Progressive glomerular sclerosis in experimental antiglomerular basement membrane glomerulonephritis. Am. J. Kidney Dis. 7:5-11.

2. Neilson, E. G., S. A. Jimenez, and S. M. Phillips. 1980. Cell-mediated immunity in interstitial nephritis. J. Immunol. 125:1708-1714.

3. McCluskey, R. T., and R. B. Colvin. 1978. Immunologic aspects of renal tubular and interstitial diseases. Annu. Rev. Med. 29:191-203.

4. Neilson, E. G., and B. Zakheim. 1983. T cell regulation, antiidiotypic immunity, and the nephritogenic immune response. Kidney Int. 24:289-302.

5. Adler, S., L. J. Striker, G. E. Striker, D. T. Perkinson, J. Hibbert, and W. G. Couser. 1986. Studies of progressive glomerular sclerosis in the rat. Am. J. Pathol. 123:553-562.

6. Striker, L. J., P. D. Killen, E. Chi, and G. E. Striker. 1984. The composition of glomerulosclerosis. Lab. Invest. 51:181-192.

7. Melcion, C., L. Lachman, P. D. Killen, L. Morel-Maroger, and G. E. Striker. 1982. Mesangial cells, effect of monocyte products on proliferation and matrix synthesis. Transplant. Proc. 14:559-564.

8. Striker, G. E., and L. J. Striker. 1985. Glomerular cells in culture. Lab. Invest. 53:122-130.

9. Kreisberg, J. I., and M. J. Karnovsky. 1983. Glomerular cells in culture. Kidney Int. 23:439-447.

10. Austin, H. A., J. H. Klippel, J. E. Balow, N. G. H. LeRiche, A. D. Steinberg, P. H. Plotz, and J. L. Decker. 1986. Therapy of lupus nephritis. N. Engl. J. Med. 314:614-619.

11. Wiggins, R. C., A. Glatfelter, and J. Brukman. 1985. Procoagulant activity in glomeruli and urine of rabbits with nephrotoxic nephritis. Lab. Invest. 53:156-165.

12. Huszar, G., J. Maiocco, and F. Naftolin. 1980. Monitoring of collagen and collagen fragments in chromatography of protein mixtures. Anal. Biochem. 105:424-429.

13. Phan, S. H., D. Schrier, B. McGarry, and R. E. Duque. 1983. Effect of the beige mutation on bleomycin-induced pulmonary fibrosis in mice. Am. Rev. Respir. Dis. 127:456-459.

14. Burton, K. 1956. A study of the conditions and mechanism of the diphenylamine reaction for the colorimetric estimation of deoxyribonucleic acid. Biochem. J. 62:315-323.

15. Phan, S. H., R. S. Thrall, and P. A. Ward. 1980. Bleomycin-induced pulmonary fibrosis in rats: biochemical demonstration of increased rate of collagen synthesis. Am. Rev. Respir. Dis. 121:501-506.

16. Lowry, O. H., N. J. Rosebrough, A. L. Farr, and R. J. Randall. 1951. Protein measurement with the folin phenol reagent. J. Biol. Chem. 193:265-270.

17. Phan, S. H., R. S. Thrall, and C. Williams. 1981. Bleomycininduced pulmonary fibrosis. Am. Rev. Respir. Dis. 124:428-434.

18. Seyer, J. M., E. T. Hutcheson, and A. H. Kang. 1976. Collagen polymorphism in idiopathic chronic pulmonary fibrosis. J. Clin. Invest. $57: 1498-1507$.

19. Phan, S. H., J. Varani, and D. Smith. 1985. Rat lung fibroblast collagen metabolism in bleomycin-induced pulmonary fibrosis. $J$. Clin. Invest. 76:241-247.

20. Furthmayr, H. 1982. Immunization procedures, isolation by affinity chromatography, and sèrological and immunochemical characterization of collagen specific antibodies. In Immuno-chemistry of the Extracellular Matrix. Vol I. Furthmayr, editor. CRC Press, Boca Raton, FL. 143-178.

21. Martinez-Hernandez, A., and P. S. Amenta. 1983. The basement membrane in pathology. Lab. Invest. 48:656-677.

22. Welgus, H. G., E. J. Campbell, Z. Bar-Shavit, R. M. Senior, and 
S. L. Teitelbaum. 1985. Human alveolar macrophages produce a fibroblast-like collagenase and collagenase inhibitor. J. Clin. Invest. 76:219-224.

23. Kovacs, E. J., and J. Kelley. 1984. Secretion of macrophage-derived growth factor during acute lung injury induced by bleomycin. $J$. Leukocyte Biol. 37:1-14.

24. Kovacs, E. J., and J. Kelley. 1985. Lymphokine regulation of macrophage-derived growth factor secretion following pulmonary injury. Am. J. Pathol. 121:261-268.

25. Phan, S. H., and R. S. Thrall. 1982. Inhibition of bleomycininduced pulmonary fibrosis by cobra venom factor. Am. J. Pathol. 107:25-28.

26. Fantone, J. C., S. L. Kunkel, and R. B. Zurier. 1985. Effects of prostaglandins on in vivo immune and inflammatory reactions. In Prostaglandins and Immunity. J. S. Goodwin, editor. Martinus Nijhoff, Boston. 123-146.
27. Eldridge, C., and R. C. Wiggins. 1987. T cells in the glomerulus, periglomerular region and around venules early in crescentic nephritis in the rabbit. Evidence for glomerulo-interstitial signals. Kidney Int. 31:318. (Abstr.)

28. Wiggins, R. C., and C. Eldridge. 1987. Monocyte/macrophage heterogeneity and migration into Bowman's space early in crescentic nephritis in the rabbit. Kidney Int. 31:333. (Abstr.)

29. Wiggins, R. C. 1985. Hageman factor in experimental nephrotoxic nephritis in the rabbit. Lab. Invest. 53:335-348.

30. Wiggins, R. C., A. Glatfelter, B. Kshirsagar, and T. Beals. 1987. Lipid microvesicles and their association with procoagulant activity in glomeruli of rabbits with nephrotoxic nephritis. Lab. Invest. 56:264272.

31. Silva, F. G., J. R. Hoyer, and C. L. Pirani. 1984. Sequential studies of glomerular crescent formation in rats with antiglomerular basement membrane-induced glomerulonephritis and the role of coagulation factors. Lab. Invest. 51:404-415. 